\title{
Glucose solvation by the ionic liquid 1,3-dimethylimidazolium chloride: A simulation study
}

\author{
T. G. A. Youngs, C. Hardacre, and J. D. Holbrey \\ Atomistic Simulation Centre, School of Maths and Physics, \\ Queen's University Belfast, \\ Belfast, BT7 1NN, UK. \\ E-mail: t.youngs@qub.ac.uk
}

September 21, 2007

\section{Supporting Information}

Here we provide the glucose $(\mathrm{OH})$-cation $(\mathrm{CH})$ radial distribution functions for reference. Also, we show various contour maps illustrating the conformational space explored by the sugar in simulations of 20 glucose molecules solvated by 100 dimethylimidazolium chloride ion pairs, as well the dependence of energetic properties on the conformation. Each graph uses conformer data from the 800,000 glucose geometries observed over four separate $5 \mathrm{~ns}$ simulations (configuration data was saved every $0.5 \mathrm{ps}$ ). 


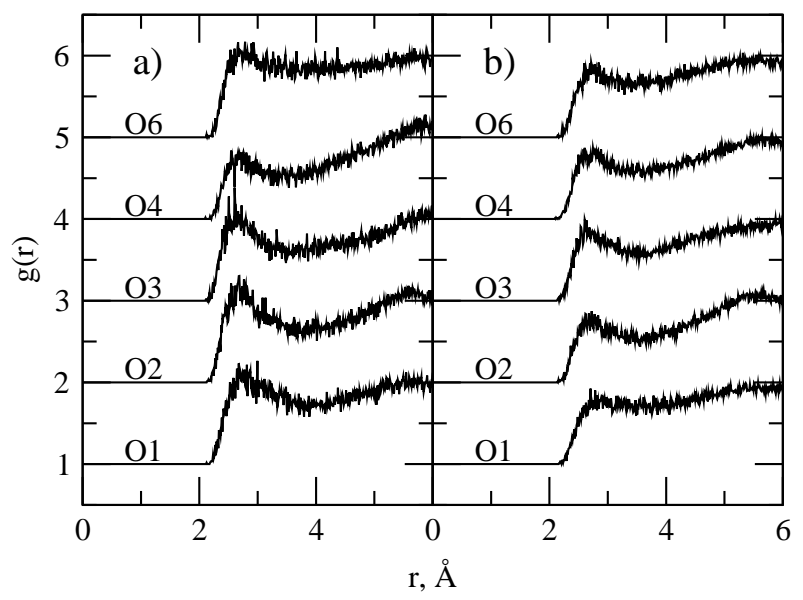

Figure 1: Site-site radial distribution functions between the hydroxyl oxygens of the sugar and a) the unique hydrogen of the imidazolium ring, and b) the two symmetry-equivalent hydrogens on the imidazolium ring (averaged).

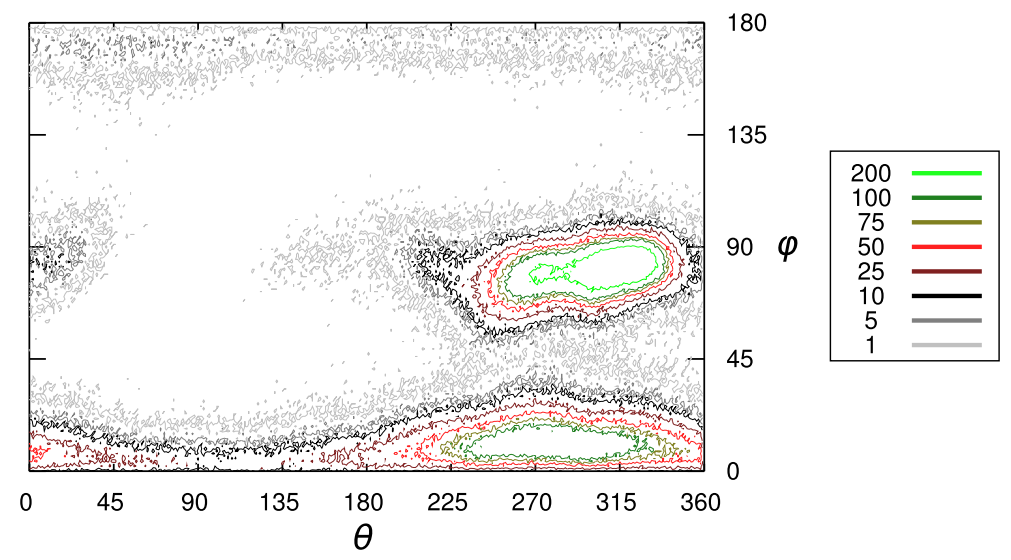

Figure 2: Distribution of spherical coordinates $\theta$ and $\phi$ for glucose geometries observed. White regions indicate that no glucose geometry matching these spherical coordinates was observed during the 20 ns of simulation time, i.e. $4 \times 5$ ns. Other colours correspond to contour levels reflecting the number of conformers of given $\theta / \phi$ observed. 


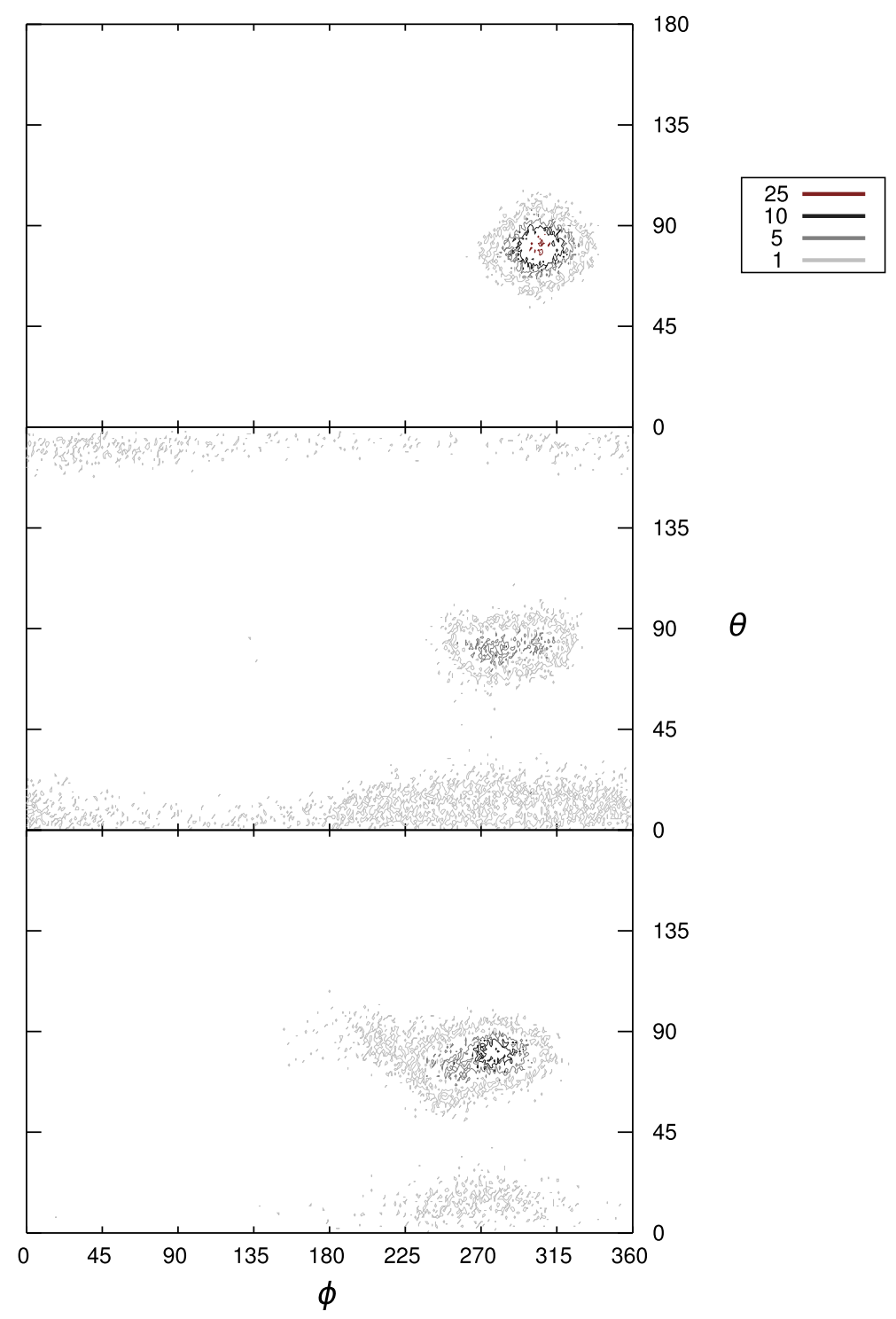

Figure 3: Distribution of spherical coordinates $\theta$ and $\phi$ for three selected glucose molecules over their respective 5 ns simulations, illustrating that glucose molecules may stay in their starting conformation for the duration of the simulation, or wander between one or more distinct regions of conformational space. 


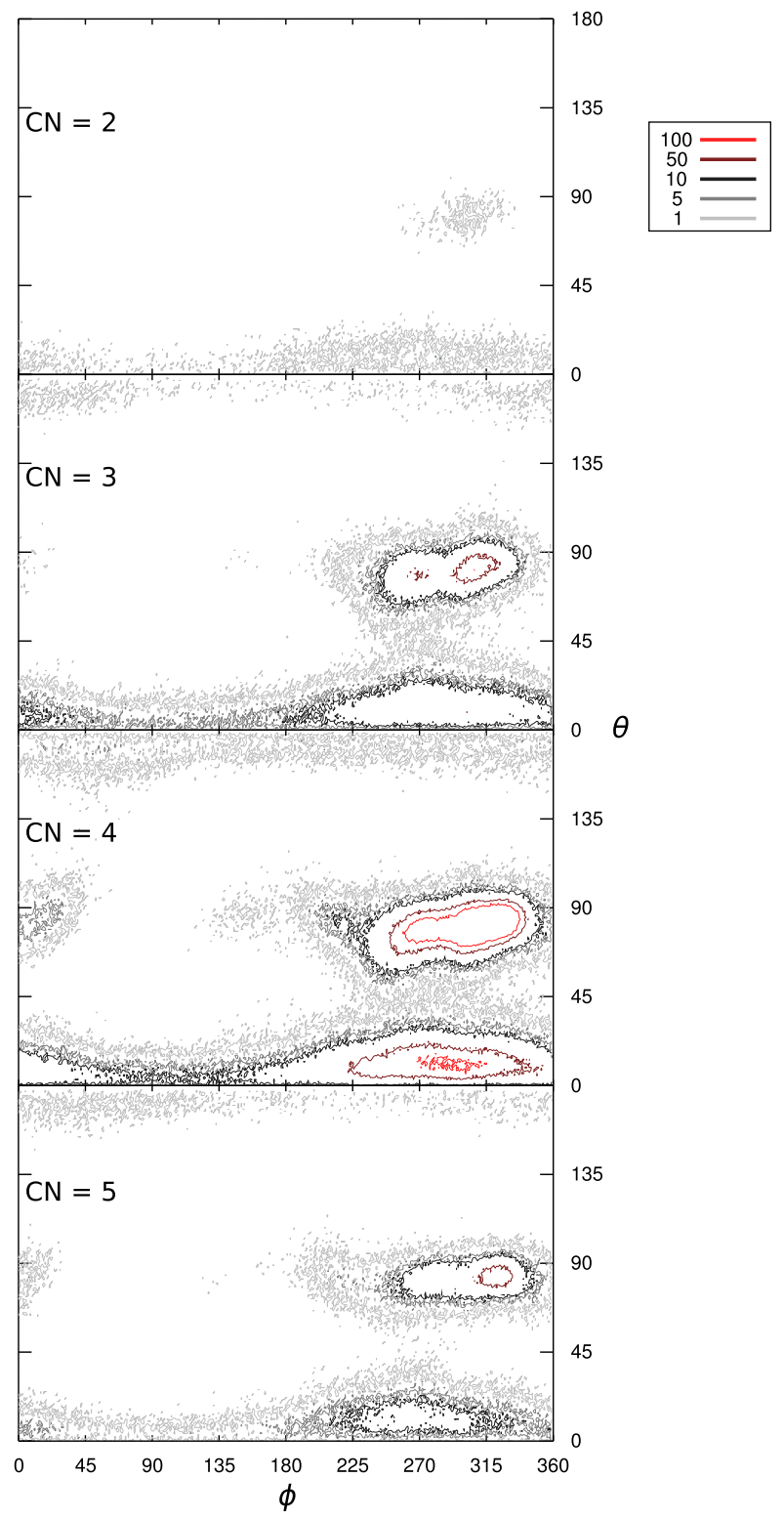

Figure 4: Distribution of spherical coordinates $\theta$ and $\phi$ over calculated chloride coordination numbers $\left(r_{c u t}=3.7 \AA\right)$ for all glucose configurations observed in the high concentration simulations. 


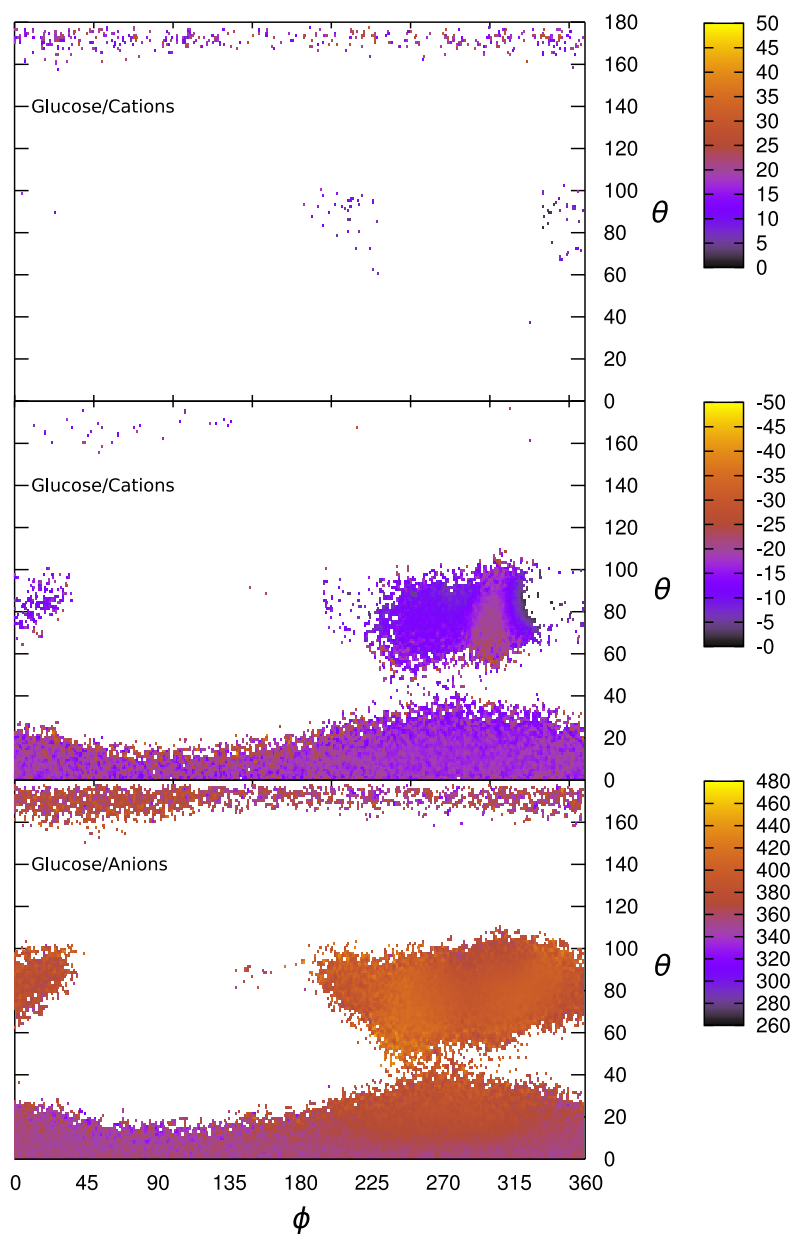

Figure 5: Contour maps of average total electrostatic interaction energies (in $\mathrm{kJ} \mathrm{mol}^{-1}$ ) between glucose molecules and ionic liquid ions (i.e. interaction energies between individual glucose molecules and all cations or anions in the system) plotted as a function of the conformation of individual sugar molecules. Interaction energies for cations are divided into positive and negative energies for clarity. No positive-energy electrostatic interactions between anions and glucose molecules were observed. 


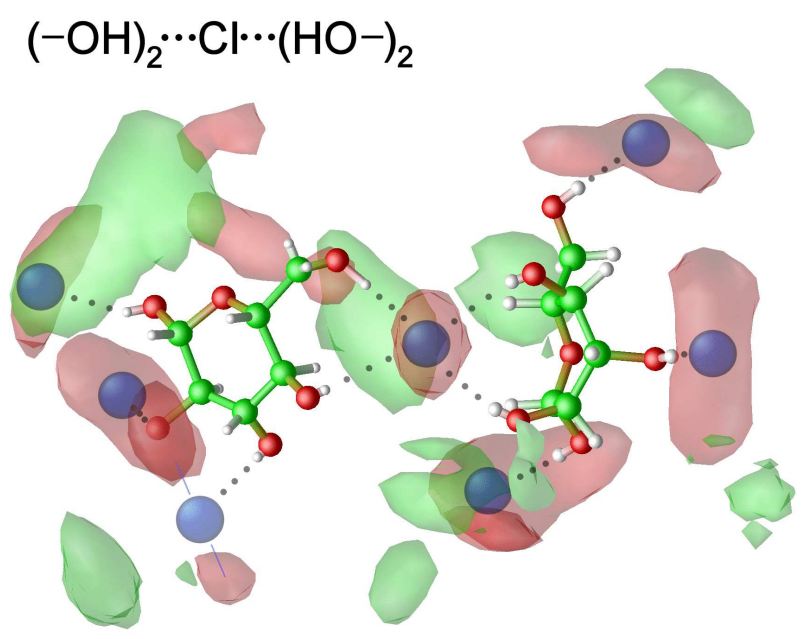

Figure 6: TOC Graphic. 